\title{
Togo - 2020 : Politique intérieure, affaires étrangères, le développement socio-économique
}

\author{
Dirk Kohnert ${ }^{1}$
}

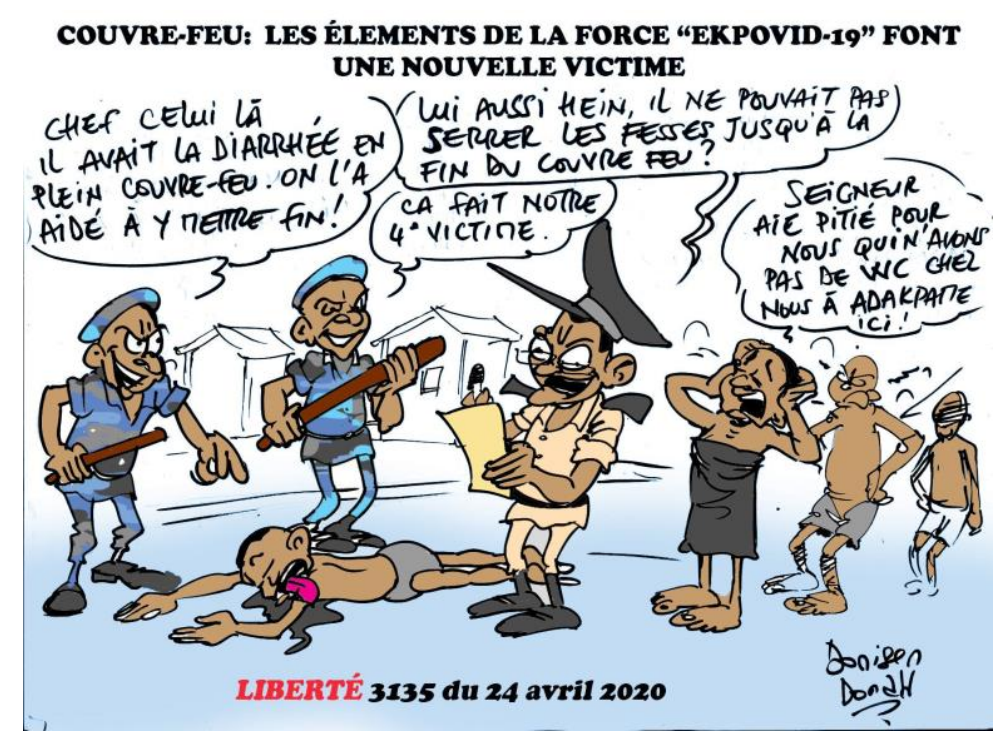

Donisen Donald, Cartoon, 2020

Résumé: Les réformes constitutionnelles et institutionnelles controversées votées en mai 2019 au Parlement ont ouvert la voie au président Gnassingbé pour se présenter pour un quatrième et un cinquième mandat parce que la loi ne s'applique pas rétroactivement. En février 2020, le président a remporté à nouveau les élections présidentielles disputées et a ainsi consolidé son pouvoir, assisté par l'armée fidèle et les services de sécurité. Le déclenchement de l'épidémie de Corona au Togo en mars et la récession économique qui a suivi a peut-être contribué à limiter les protestations populaires contre le régime de Gnassingbé. Le bilan du gouvernement en matière de droits de l'homme s'est amélioré, mais reste médiocre. Pourtant, la communauté internationale a suivi une approche de «laissez-faire» dans l'intérêt de la stabilité régionale. L'économie est entrée en récession en raison des effets négatifs économiques mondiaux de la crise corona. L'indice de démocratie de l'Economic Intelligence Unit, à Londres, classait toujours le Togo comme un «régime autoritaire»..

Mots-clé: étude nationale, Togo, Afrique de l'Ouest, politique intérieure, affaires étrangères, développement socio-économique, études africaines, CEDEAO, UEMOA

JEL-codes: F35, N97, O17, O55, Z13

\footnotetext{
${ }^{1}$.Expert associé à l'Institut des Affaires Africaines, Institut Allemand d'Études Mondiales et Régionales (GIGAHambourg). Brouillon: Janvier 8, 2021.
} 
Version française étendue de l'auteur de : Kohnert, D. (2022): Togo. In : Albert K. Awedoba / Benedikt Kamski / Andreas Mehler / David Subudubudu (eds.), Africa Yearbook. Vol. 17 - Politics, economy and society South of the Sahara in 2020. Leiden \& Boston: Brill:, à paraitre

\section{Politique intérieur}

Les élections présidentielles contestées ont dominé la scène politique nationale au Togo dès le début de l'année. Le gouvernement n'avait pas fixé la date des élections que peu de temps auparavant, le 6 décembre 2019, au 22 février 2020. Il ouvrait ainsi la voie à un quatrième et finalement cinquième mandat quinquennal pour Faure Gnassingbé en 2020 et 2025. Cela contredit la limitation de mandats présidentiels à deux mandats consécutifs dans la constitution de 1992. Ce dernier, cependant, avait été modifié lors d'un «coup d'État constitutionnel » de 2002 et à nouveau par les réformes constitutionnelles et institutionnelles controversées de mai 2019 qui ont ouvert la voie au président Gnassingbé pour se présenter pour un quatrième et un cinquième mandat parce que la loi ne s'appliquait pas rétroactivement. En outre, la réforme de 2019 a changé le système électoral de la présidence en un système à deux tours, remplaçant l'ancien système uninominal à un tour et accordant au président l'immunité à vie pour les mesures prises pendant son mandat.

Au total, dix candidats avaient été acceptés pour les élections présidentielles par la Cour constitutionnelle telle que announcer le 17 janvier. Outre le président sortant Faure Gnassingbé et le chef du plus grand parti d'opposition, l'Alliance nationale pour le changement (ANC), Jean-Pierre Fabre, ce comprenait également Agbéyomé Kodjo, du Mouvement patriotique pour la démocratie et le développement (MPDD). Il avait déjà été Premier ministre sous feu Eyadéma Gnassingbé. Capitalisant sur la désunion de l'opposition, Kodjo, soutenu par certains partis de la coalition d'opposition C14 ainsi que par l'archevêque en retraite de Lomé Mgr Phillippe Kpodzro, a contesté la direction de l'opposition par Jean-Pierre Fabre. Kpodzro avait toujours eu une grande place dans la société civile togolaise en raison de son rôle de médiateur lors du renouveau démocratique du Togo au début des années 1990. La candidature de Kodjo a plongé l'opposition encore plus au point d'éveiller la suspicion d'agir dans l'intérêt des pouvoirs au pouvoir.

Par conséquent, un nouveau mouvement politique d'opposition, " Dynamique Mgr Kpodzro »(DMK), une association de partis d'opposition et d'organisations de la société civile, a été formé par des partisans de l'archevêque Kpodzro, pour soutenir Kodjo pour les présidentielles du 22 février.

Le 1er février 2020, les organisations de défense des droits civiques et les groupes d'opposition ont manifester et exigé que les résultats régionaux et locaux des circonscriptions soient publiés directement et non plus de manière centralisée par la Commission électorale nationale indépendante (CENI) afin de réduire la possibilité de fraude électorale. Pourtant, le ministre des droits de l'homme, Christian Trimua, a annoncé que le gouvernement n'approuvait pas cette demande et que seuls les résultats agrégés seraient publiés. La CENI a été jugée partiale par l'opposition parce que, entre autres, seuls deux de ses 19 membres ont été attribués par l'opposition et parce que les commissions électorales régionales, chargées de soumettre les résultats à la CENI, étaient dirigées par des juges choisis par le parti au pouvoir.

La campagne électorale a commencé le 6 février, deux semaines avant le scrutin et s'est terminée 24 heures avant l'ouverture des bureaux de vote. Environ 10.000 soldats ont 
Version française étendue de l'auteur de : Kohnert, D. (2022): Togo. In : Albert K. Awedoba / Benedikt Kamski / Andreas Mehler / David Subudubudu (eds.), Africa Yearbook. Vol. 17 - Politics, economy and society South of the Sahara in 2020. Leiden \& Boston: Brill:, à paraitre

été déployés pour sécuriser les élections, une perspective qui, selon les militants de l'opposition, équivalait à de l'intimidation.

L'élection a été surveillée par 315 observateurs électoraux internationaux, principalement de l'ONU, de l'Union africaine (UA) et de la Communauté économique des États de l'Afrique de l'Ouest (CEDEAO). En outre, chaque candidat a été autorisé à envoyer des représentants dans près de 9.400 bureaux de vote. Mais le 17 janvier, le gouvernement a annulé les pouvoirs d'observateur électoral de la plus grande organisation indépendante de la société civile, la Concertation nationale de la société civile, ainsi que ceux des observateurs de l'Église catholique. Par ailleurs, le National Democratic Institute (NDI, Washington), reconnu internationalement pour son soutien aux élections démocratiques en Afrique depuis des décennies, a vu son accréditation retirée et l'un de ses collaborateurs expulsé du Togo sans avertissement. De plus, l'accès au site Web NDI et à WhatsApp a été bloqué par le gouvernement. L'UE n'a pas surveillé les élections, surtout parce que le gouvernement de Lomé n'avait pas honoré les recommandations des missions d'observation électorale de l'UE dans le passé. Un système électronique spécialement créé pour enregistrer les résultats des élections a été renversé par l'autorité électorale deux jours avant l'élection. Le jour du scrutin, l'accès à Internet a été restreint et les réseaux sociaux, ainsi que les sites Web médiatiques critiques, ont été bloqués.

Le nouveau code électoral de mai 2019 et l'autorisation des votes de la diaspora par le Parlement le 5 novembre 2019 ont permis aux Togolais de la diaspora (estimée à 2 millions de personnes) de voter, la première fois dans l'histoire du pays. Cependant, la CENI n'avait enregistré que 212.538 citoyens togolais de la diaspora ayant le droit de vote. Le 13 novembre 2019, le gouvernement avait décidé que la diaspora togolaise, représentée par les 77 membres du nouvellement créés Haut Conseil togolais des affaires étrangères (HCTE), organisé par le gouvernement à Lomé, ne pouvait voter que dans six pays sélectionnés : France, États Unis, République démocratique du Congo (RDC), Gabon, Nigéria et Maroc. Les nombreux réfugiés togolais vivant en Allemagne par exemple, qui avaient développé une position particulièrement critique contre le régime au pouvoir parce que la plupart d'entre eux avaient été politiquement persécutés par la dictature de Gnassingbé dans les années 1990, se sont vu refuser le droit de vote. En outre, la disposition d'application garantit que pratiquement aucun électeur d'opposition n'oserait s'inscrire pour voter dans son ambassade. Enfin, seuls 348 citoyens de la diaspora y ont participé.

Selon la Commission électorale nationale (CENI), il y avait 3.738.786 électeurs inscrits dont 2.769.286 se sont rendus aux urnes selon les résultats préliminaires. La CENI a dénombré 983.413 votes blancs et nuls. Selon les résultats définitifs officiels, confirmés le 3 mars par la Cour constitutionnelle biasée, dont le peut glorieux président Abdou Assouma avait été reconduit peu avant le 30 décembre 2019, le président sortant Faure Gnassingbé a remporté les élections avec $70,78 \%$, soit 1.760 .309 voix, déjà au premier tour, contre 19,46\%, soit 483.926 voix, pour son concurent Agbeyome Kodjo qui est arrivé deuxième.

L'ancien chef de l'opposition, Jean-Pierre Fabre de l'ANC, a terminé loin derrière à la troisième place avec 4,68\%, soit 116336 voix. Le fort taux de participation contesté de $76,62 \%$, soit 15 points de plus qu'en 2015, semble prouver l'intérêt permanent du grand 
Version française étendue de l'auteur de : Kohnert, D. (2022): Togo. In : Albert K. Awedoba / Benedikt Kamski / Andreas Mehler / David Subudubudu (eds.), Africa Yearbook. Vol. 17 - Politics, economy and society South of the Sahara in 2020. Leiden \& Boston: Brill:, à paraitre

public pour les élections multipartites malgré l'organisation biaisée du processus électoral.

Kodjo, soutenu par l'opposition, a déjà déclaré les résultats préliminaires des élections comme un faux. Soutenu par deux autres candidats moins importants, Gogué et Wolou, il soutient avoir obtenu la majorité des voix. Ils ont appelé le président Gnassingbé à démissionner en raison de tentatives d'intimidation et de graves fraudes électorales. L'évêque Kpodzro, qui soutenait Kodjo, a qualifié le résultat provisoire de farce et a appelé la population à manifester. Cependant, l'appel de Kodjo contre les résultats devant la Cour constitutionnelle a été rejeté. Au contraire, les observateurs de l'UA et de la CEDEAO ont félicité le peuple togolais pour le bon déroulement et le déroulement pacifique des élections. En mars, l'immunité de l'ancien Premier ministre Agbeyome Kodjo a été levée par le Parlement car il maintenait toujours sa victoire. Peu de temps après, il a été arrêté pour ne pas avoir comparu devant le service central de recherches et d'enquêtes criminelles de la gendarmerie du pays après s'être proclamé « président élu du Togo » le 21 avril.

Les évêques catholiques du Togo ont condamné l'arrestation du chef de l'opposition dans un mouvement qui a inspiré des tensions post-électorales persistantes dans le pays. Cependant, l'éclosion de l'épidémie de Corona au Togo en mars 2020 avec 3.604 cas confirmés et 68 décès jusqu'au 29 décembre et la récession économique qui a suivi principalement due à des chocs externes - ont peut-être contribué à limiter les protestations populaires contre le régime de Gnassingbé.

Parce que le DMK était perçu comme une menace potentielle pour les pouvoirs régnantes, il était visé par les services de sécurité du régime. Certains de ses principaux membres, dont l'avocate et militante des droits de l'homme, Brigitte Kafui AdjamagboJohnson, première femme à se présenter aux élections présidentielles au Togo en 2010, et Gérard Yaovi Djossou (orthographe alternative, Djoussou) ont été emprisonnés en raison d'une attaque présumée contre la sécurité intérieure de l'Etat les 27 et 30 novembre. Djossou, responsable des droits de l'homme et des questions sociales au sein du DMK, avait été arrêté dans la rue à Hanoukopé, Lomé, après avoir rencontré des ambassadeurs du soi-disant «Groupe des Cinq »- l'États-Unis, France, Allemagne, l'UE et le Programme des Nations Unies pour le développement. Il aurait été violemment battu et aurait subi de nombreuses heures d'interrogatoire, menotté et sans accès à son avocat. Adjamagbo-Johnson a été arrêtée à son tour lorsqu'elle est allée lui rendre visite. Le 4 décembre, les deux accusés ont été inculpés de «complot criminel et atteinte à la sécurité intérieure ». Ils ont été libérés provisoirement le 17 décembre après près de trois semaines de détention. Déjà auparavant, le 21 avril, environ 16 membres du DMK qui avaient protesté contre l'arrestation du politicien de l'opposition Agbéyomé Kodjo le même jour, ont été arrêtés puis reconnus coupables de «rébellion » et de «complicité dans la rébellion » et condamnés à un an en prison, dont huit mois avec sursis. Ils ont été libérés le 25 août.

En raison de la pandémie de Covid-19, divers droits civiques ont été limités. La pandémie a débuté le 6 mars, déclenchée par une Togolaise vivant à Lomé, qui était restée 11 jours en France, en Turquie et au Bénin avant la confirmation de sa contamination au Covid-19. Afin de contrôler la propagation du virus au Togo, toutes les frontières du pays ont été fermées et une quarantaine a été appliquée aux villes de 
Version française étendue de l'auteur de : Kohnert, D. (2022): Togo. In : Albert K. Awedoba / Benedikt Kamski / Andreas Mehler / David Subudubudu (eds.), Africa Yearbook. Vol. 17 - Politics, economy and society South of the Sahara in 2020. Leiden \& Boston: Brill:, à paraitre

Lomé, Tsévié, Kpalimé et Sokodé le 20 mars pendant deux semaines. Jusqu'en septembre, il y avait 1.795 cas confirmés. Le bilan est passé à 48 morts. Le 16 mars, le gouvernement a annoncé un fonds de 2 milliards de F CFA pour lutter contre la pandémie. Il a également suspendu les vols en provenance d'Italie, de France, d'Allemagne et d'Espagne et a annulé tous les événements internationaux pendant trois semaines; obliger les personnes qui se trouvaient récemment dans un pays à haut risque à s'auto-isoler. À partir du 14 septembre 2020, tous les voyageurs au Togo doivent installer l'application « Togo Safe » à leur arrivée sur le territoire.

La pandémie de Covid-19 a également gravement affecté les activités des Organisatins de la Société Civile (OSC). Les défis les plus courants ont été la perte de financement, qui a mis les opérations au point mort. La réduction du financement a également conduit à des changements opérationnels et structurels majeurs et à l'incapacité de mettre en œuvre les activités du programme en raison des mesures préventives du gouvernement contre la pandémie.

Lors des élections municipales de juin 2019, qui avaient été reportées à plusieurs reprises depuis 1987, de nouvelles maires avaient été élues dans 117 villes et municipalités nouvellement désignées, dont 20 Mme la maires. Parce qu'il n'y avait pas de subventions et donc pas de compensation pour les maires après dix mois de mandat; les relations entre préfets et maires étaient tirées au couteau dans certaines préfectures. Le 7 septembre 2020, le ministre de l'Administration territoriale Payadowa Boukpessi a annoncé que l'Etat procéderait dans les semaines à venir au versement d'allocations au titre du Fonds d'Appui aux Collectivités Locales (FACT), estimé à environ 3 milliards F CFA.

Le 28 septembre 2020, une nouvelle Première ministre, Victoire Tomegah Dogbe (61 ans, née le 23 décembre 1959) a été nommée Premier ministre. Elle a été la première femme à occuper le poste. De formation économiste, Mme Dogbe était ministre du Développement à la base, de l'Artisanat, de la Jeunesse et de l'Emploi des Jeunes ainsi que Directrice de cabinet du Président Gnassingbé depuis 2010. Elle a nommé un nouveau gouvernement avec un record de $30 \%$ des 33 postes ministériels attribués aux femmes, y compris Essozimna Marguerite Gnakade au poste de ministre de la Défense la première fois qu'une femme occupe ce poste. En général, le Premier ministre a davantage besoin du soutien du président que de celui du parlement s'il veut mettre en œuvre des politiques publiques importantes.

Le 19 octobre 2020, le président a réorganisé son équipe de proches collaborateurs. Une « armoire de cuisine » d'un nouveau garde de conseillers jeunes et dynamiques, dont un officier de liaison de l'armée, a été formé pour assister le Premier ministre. Il était destiné à compléter la vieille garde des conseillers spéciaux du président ${ }^{2}$. Les membres de la nouvelle « armoire de cuisine » comprenaient les nouveaux conseillers suivants :

\footnotetext{
${ }^{2} \mathrm{La}$ « vieille garde »' des conseillers spéciaux comprenait le frère de Faure, Amah Gnassingbé, deuxième vice-président de l'ancien parti de l'UFC d'opposition, qui avait accepté en 2006 un poste au gouvernement en tant que ministre d'État, mais aussi le célèbre avocat français Charles Debbasch, qui aurait joué un rôle crucial dans le « coup d'État constitutionnel » par lequel Faure Gnassingbé a succédé à son père Eyadéma, ainsi que Barry Moussa Barqué et Koffi Sama, En octobre 2020, Carlos Lopes, aurait été l'un des principaux architectes du Plan national de développement du Togo (PND) a rejoint l'équipage de hauts conseillers retraités de réputation internationale de Faure Gnassingbé, comme Tony Blair,
} 
Version française étendue de l'auteur de : Kohnert, D. (2022): Togo. In : Albert K. Awedoba / Benedikt Kamski / Andreas Mehler / David Subudubudu (eds.), Africa Yearbook. Vol. 17 - Politics, economy and society South of the Sahara in 2020. Leiden \& Boston: Brill:, à paraitre

- D'abord, Ablamba Sandra Johnson, elle a le rang de ministre en tant que nouveau secrétaire général de la présidence et a remplacé Patrick Daté TéviBenissan, décédé le 2 septembre. Elle dirige également le cabinet civil du chef de l'Etat depuis Victoire Tomegah Dogbe a été promu au bureau du Premier ministre. Ainsi, elle a été récompensée pour avoir contribué au succès des réformes qui ont permis au Togo des progrès spectaculaires dans les classements 2019 et 2020 des rapports «Doing Business» de la Banque mondiale. Ce dernier avait été renforcé par l'Indice Ibrahim de la gouvernance africaine 2020 qui comptait le Togo parmi les cinq meilleurs améliorateurs au cours de la dernière décennie pour la gouvernance globale (30e sur 54 en 2019; score 49,1, +5,2, depuis 2008)

- Deuxièmement, Kouessan Joseph Yovodevi, nouveau directeur de la communication à la présidence. Ce journaliste, formé à l'Institut supérieur de la presse à Lomé, il devrait restructurer la communication gouvernementale.

- Troisièmement, Komlan Adjitowou a pris la tête du cabinet militaire du Palais. Il était auparavant chef d'état-major adjoint des forces armées togolaises, élevé au grade de général en 2018. Il succède au colonel Awoki Panassa nommé, en janvier 2020, ambassadeur du Togo au Ghana. Dans ce poste, Adjitowou servira d'interface entre le président et les forces de défense.

- Quatrièmement, Djibril Mohaman Awalou, en tant que coordinateur national pour la gestion de la réponse au Covid-19. Il est professeur d'université et directeur central du service de santé de l'armée et de la clinique médicale et chirurgicale (prétendument proche des militaires) de la clinique du CHU Sylvanus-Olympio à Lomé. Awalou rend compte quotidiennement à Faure Gnassingbé, qui est à la tête du comité de crise de lutte contre la pandémie.

Des troubles mijotés au sein des services de sécurité ont alarmé le gouvernement ainsi que le grand public. Après une vague sans précédent de désertions d'officiers des forces armées togolaises (FAT) déjà début 2018, et de trois autres militaires se suicidant en avril et juin 2018, phénomène à peine inconnu jusque-là, il s'en est suivi une autre incidence inquiétante en 2020. Le 4 mai 2020, le colonel Bitala Madjoulba, commandant du 1er bataillon d'intervention rapide (BIR) a été assassiné, abattu avec son revolver dans son bureau. Le fait que ce crime concernait un haut fonctionnaire chargé de la protection des hauts fonctionnaires de l'État a fait de cet assassinat une affaire d'État. Un jour seulement après l'assassinat, Faure Gnassingbé a nommé le lieutenant-colonel Atafai Tchangani pour le remplacer. Ce dernier était chef de section à l'École spéciale militaire de Saint-Cyr (ESM, première académie militaire française) et commandant de l'opération des Nations Unies en Côte d'Ivoire (bataillon de l'ONUCI).

Dominique Strauss-Khan et Lionel Zinsou. Lopes, est un économiste du développement bissau-guinéen et ancien secrétaire exécutif de la Commission économique des Nations Unies pour l'Afrique (CEA, 20122016). Par ailleurs, en juin 2020, Lotfi Bel Hadj, essayiste, économiste et homme d'affaires francotunisien, est devenu le communicateur du Togo. Après avoir vu une partie de ses opérations de marketing politique en ligne dévoilées le mois dernier par le célèbre think-tank américain Atlantic Council, le communicateur franco-tunisien a signé des contrats de communication et d'influence avec la présidence togolaise. 
Version française étendue de l'auteur de : Kohnert, D. (2022): Togo. In : Albert K. Awedoba / Benedikt Kamski / Andreas Mehler / David Subudubudu (eds.), Africa Yearbook. Vol. 17 - Politics, economy and society South of the Sahara in 2020. Leiden \& Boston: Brill:, à paraitre

Le 7 mai, trois organisations des droits de l'homme - la Ligue togolaise des droits de l'homme (LTDH), l'Association des victimes de l'impunité au Togo (Asvito) et la Ligue des consommateurs du Togo (LCT) - avaient interpellé la famille du lieutenant-colonel Bitala Madjoulba à porter plainte contre l'Etat togolais, censé garantir la sécurité et l'intégrité des personnes sur son territoire. La commission d'enquête mise en place par le gouvernement en mai, dirigée par le ministre de la Sécurité et de la Protection civile, le général de brigade Yark Damehame, n'a révélé aucun résultat. Aussi, un groupe d'avocats a saisi le Conseil des droits de l'homme des Nations unies (CDH) à Genève le 10 juillet pour faire «la pression internationale » sur l'Etat togolais, afin que «justice soit faite $\gg$.

Jusqu'à la fin de l'année, le gouvernement n'a publié aucune déclaration officielle sur ce meurtre. Cependant, le 3 octobre, le président Gnassingbé a procédé à plusieurs nominations par décret, au sein des Forces armées togolaises (FAT). Le général de brigade Komlan Adjitowou a été nommé chef d'état-major du président de la République. Son ancien poste de chef d'état-major de la FAT sera désormais occupé par le colonel Kodjo Ekpe Apedo. Le colonel Kassawa Kolémaga, ancien directeur des opérations des FAT et ancien chef de l'Agence nationale de renseignement (ANR) a été promu chef d'état-major de l'armée. Le colonel Tassounti Djato, jusqu'alors commandant de la base de chasse de Niamtougou (BCN, 350 personnes) à Niamtougou, chef-lieu de la préfecture de Doufelgou dans la région de Kara, région natal et fief de la famille Gnassingbé, est nommé chef d'état-major de l'armée de l'air. Enfin, l'armée de l'air aura le colonel Toussounti Djato comme nouveau patron; il était en charge de la base aérienne de Niamtougou (nord). Pour rappel, le chef d'état-major général des forces armées togolaises est le général de brigade Félix Abalo Kadhanga, alors que le ministère des défenses reste aux mains de Faure Gnassingbé pour empêcher toute tentative de coup d'État.

La violation continue des droits de l'homme au Togo demeure une préoccupation particulière de la communauté internationale. Selon un rapport d'Amnesty International sur la situation des droits humains au Togo, présenté au Comité des droits de l'homme des Nations Unies (du 2 au 27 mars), la situation des droits humains au Togo s'est fortement dégradée en raison des tensions politiques et sociales croissantes liées à les perspectives de candidature du chef de l'Etat pour un quatrième et cinquième mandat et les réformes constitutionnelles contestées de 2019. De plus, le Togo se classe toujours parmi les États les plus corrompus au monde (rang 130 sur 180 pays (Note: 29 sur 100; IPC-2020). Notamment, le niveau élevé des flux financiers illicites (FFI) qui est fortement corrélé au blanchiment d'argent suscité l'inquiétude de la communauté internationale des donateurs.

L'empiètement croissant sur la liberté des médias et sur l'Internet a pris diverses formes, allant de l'augmentation subtile des pouvoirs de réglementation sur les médias sociaux aux fermetures d'Internet à l'échelle du pays. En juin, la HAAC a suspendu le bimensuel «Panorama » pour violation des règles professionnelles du journalisme et un rapport critique sur le chef de l'État en mai. Le 25 mars, la HAAC a retiré l'autorisation du journal «La Nouvelle » de publier des « informations non vérifiées », et l'incitation à la haine ethnique et religieuse. Le 4 novembre, le directeur de la revue d'opposition « L'Alternative », Ferdinand Ayité, ainsi que la revue elle-même ont été condamnés chacun à une amende de deux millions de F CFA pour diffamation dans l'affaire 
Version française étendue de l'auteur de : Kohnert, D. (2022): Togo. In : Albert K. Awedoba / Benedikt Kamski / Andreas Mehler / David Subudubudu (eds.), Africa Yearbook. Vol. 17 - Politics, economy and society South of the Sahara in 2020. Leiden \& Boston: Brill:, à paraitre

Pétrolegate. Ayité avait accusé le gérant du Comité de suivi des fluctuations des prix des produits pétroliers, Fabrice Adjakly, d'être responsable de détournement de $500 \mathrm{~F}$ CFA Mrd. Dans la nuit du 29 décembre, Carlos Kétohou, de «l'Indépendant Express », a été arrêté par des hommes lourdement armés du Service central d'enquête et d'enquête criminelle (SCRIC), une unité spéciale de la gendarmerie nationale. Deux jours plus tard, des organisations de presse togolaises comme OTM, CONAPP, PPT, URATEL, ATOPPEL, UJIT et SYNJIT condamnent l'arrestation qui, selon eux, équivaut à un « enlèvement ».

En outre, l'ingérence du gouvernement dans l'Internet et les téléphones portables était particulièrement préoccupante dans le contexte des approches de développement participatif des organismes donateurs internationaux, étant donné la dépendance croissante aux technologies numériques de l'information et de la communication (TIC). Par conséquent, l'indice de démocratie EIU 2019 (publié le 31 janvier 2019) a classé les médias togolais dans l'ensemble comme «non libres », alors que Freedom House (2020) au contraire avait relevé le statut général du Togo, y compris le statut de la liberté de la presse, depuis 2014 de « gratuit » à « partiellement gratuit », avec un statut global de 47 (sur 100) déjà en 2018.

Par ailleurs, le gouvernement a dû accélérer la mise en œuvre des recommandations de la Commission Vérité, Justice, Réconciliation (CVJR, Plateforme citoyenne justice et vérité, PCJV) créée pour compenser l'impunité des membres des services de sécurité pendant la dictature d'Eyadéma. En 2020, le HCRRUN a contacté des personnes ayant subi des dommages dans les années 1990 dans le cadre de la protection de la faune, et de l'incident de Barkoissi entre Anoufo et Moba, pour mettre en œuvre les réparations préconisées par la CVJR dont l'objectif était de parvenir à un meilleur vivre ensemble entre les communautés. La mise en œuvre des recommandations de la CVJR dans cette partie du Togo s'est étalée sur plusieurs jours dans différentes localités (Tône, Cinkassé, Mandouri, Kpendjal etc) pour respecter les mesures conservatoires dans le cadre de la lutte contre Covid-19. Près de 35.000 personnes, victimes de violences sociopolitiques de 1958 à 2005, ont été identifiées dans tout le Togo.

Le statut général du Togo dans la cote «Liberté dans le monde » de Freedom House en 2020 est resté « partiellement libre » comme les deux années précédentes. Dans l'indice de démocratie 2019 de l'Economist Intelligence Unit, le Togo est passé du rang 130 (2015, 3,32 sur 10 points) au rang 126 (2019; 3,30) sur 167 pays, principalement en raison de sa culture politique (5 sur 10). Pour le reste, l'EIU a qualifié le Togo de « régime autoritaire » comme auparavant.

\section{Affaires étrangères}

En janvier (17 janvier), les cinq chefs d'État du Congo-Brazaville, de l'Ouganda, du Sénégal, de la Gambie et du Togo se sont réunis à Lomé pour un sommet contre le trafic de drogue. Le trafic de faux médicaments tuerait environ 900.000 Africains chaque année, dont 120.000 enfants de moins de cinq ans. Ainsi, plus d'Africains meurent à cause de faux médicaments que de malaria. Le sommet avait été organisé en partenariat avec l'ONG Brazzaville, une fondation axée sur la résolution des conflits et la protection 
Version française étendue de l'auteur de : Kohnert, D. (2022): Togo. In : Albert K. Awedoba / Benedikt Kamski / Andreas Mehler / David Subudubudu (eds.), Africa Yearbook. Vol. 17 - Politics, economy and society South of the Sahara in 2020. Leiden \& Boston: Brill:, à paraitre

de l'environnement. Deux autres pays concernés étaient le Niger et le Ghana. Le président du Niger, Mahamadou Issoufou, n'a pas participé, compte tenu de la situation sécuritaire précaire dans son pays, tandis que son homologue ghanéen, Nana Akoufo$\underline{\text { Addo, }}$ était représenté par son ministre de la Santé.

Bien que la production de cannabis au Togo soit illégale, le Togo compte parmi les dix pays africains ayant une production importante de cannabis, à savoir la RCA, la RDC, la Gambie, le Ghana, la Côte d'Ivoire, le Malawi, le Nigéria, la Sierra Leone, le Togo et l'Ouganda selon l'indice de la criminalité organisée en Afrique (OCI) Togo. Le 17 mai, les douanes togolaises ont intercepté à Tchalo (au sud de Sokodé), 1.042 pains de cannabis cachés dans une voiture charger de banane. La plupart du cannabis est destiné au commerce, la population locale n'en consommait qu'à $1 \%$.

En février, des représentants de Microsoft ont révélé lors d'un Cyber Africa Summit à Lagos (25 au 26 février) que le secteur financier des pays africains était devenu l'une des cibles privilégiées des hackers. Ainsi, Cyber Defence Africa, un partenariat publicprivé entre la société polonaise Asseco Data Systems SA (ADS) et l'agence togolaise de cybersécurité (Ancy) avait déjà été créée en février 2019, comme annoncé lors du dernier forum Africa CEO à Kigali en mars 2019.

Le 26 février, le gouvernement américain a publié une critique remarquablement franche sur le déroulement des élections présidentielles de 2020 au Togo. En particulier, il a désapprouvé les petits efforts pour l'observation indépendante des élections, notamment la décision de la CENI de révoquer l'accréditation du National Democratic Institute (NDI) à Washington D.C. et l'expulsion de ses observateurs trois jours avant les élections. Washington a appelé Lomé à publier les résultats des élections par bureau de vote et pas seulement les résultats agrégés pour permettre des vérifications croisées.

En raison du poids économique de la pandémie Corona, les chefs d'État de l'Union économique et monétaire ouest-africaine (UEMOA) ont déclaré une suspension temporaire du pacte de croissance et de stabilité de l'UEMOA (fixant six critères de convergence, dont 3\% de limite du déficit budgétaire du PIB). En conséquence, les pays membres ont été autorisés à augmenter temporairement leur déficit budgétaire global et à utiliser tout soutien extérieur supplémentaire que les donateurs pourraient fournir en réponse à la crise Covid. Par ailleurs, un Fonds de solidarité nationale et économique de 400 milliards de FCFA (11,8\% du PIB) a été créé le 1er avril pour être financé par le gouvernement, des partenaires internationaux et des particuliers. Peu avant, le 20 mars, l'UEMOA a annoncé un plan d'action fortement tributaire du financement des partenaires au développement. Le financement global nécessaire est estimé à 70 milliards F CFA (environ 130 millions USD ou 2\% du PIB). Néanmoins, la monnaie CFA n'a pas encore été affectée en raison d'un retrait des capitaux étrangers provoqué par une pandémie, d'une augmentation du déficit du compte courant ou de la croissance des agrégats monétaires due aux prêts préférentiels, et des tendances inflationnistes significatives n'ont pas été décelées.

La pandémie de Covid a également affecté, entre autres, le commerce transfrontalier informel des petits commerçants profondément enraciné dans la region. Le 8 avril, environ 90 citoyens béninois et togolais, dont 68 hommes et 22 femmes, ont été arrêtés dans la région ghanéenne d'Ashanti parce qu'ils avaient tenté d'entrer illégalement dans 
Version française étendue de l'auteur de : Kohnert, D. (2022): Togo. In : Albert K. Awedoba / Benedikt Kamski / Andreas Mehler / David Subudubudu (eds.), Africa Yearbook. Vol. 17 - Politics, economy and society South of the Sahara in 2020. Leiden \& Boston: Brill:, à paraitre

le pays en contournant les mesures de verrouillage de la covid. Ils ont ensuite été placés en isolement à l'hôpital Atonsu Agogo. Les autorités ont alerté la population locale dans les régions frontalières pour qu'elle surveille d'autres migrants illégaux en provenance des États voisins. À peine un mois plus tard, le 7 mai, le Service de l'immigration du Ghana (GIS) a révélé qu'il avait attrapé plus de 100 commerçants d'Accra au cours des trois dernières semaines, pour la plupart des femmes, qui se sont rendus à Aflao à la frontière ghanéenne, où ils sont restés à divers endroits, attendant leur tour que les opérateurs «okada» les aident à traverser le Togo dans l'espoir de passer à Lomé par des itinéraires non approuvés, en violation de la fermeture de la frontière.

Le 24 avril, le gouvernement a annoncé l'achèvement de l'adhésion du pays à l'Agence africaine d'assurance commerciale (ATI), devenant ainsi le 8e actionnaire souverain africain des 18 pays membre actuels. ATI a été fondée en 2001 par les États africains, principalement du COMESA et d'actionnaires d'entreprises comme la Banque africaine de développement, la Banque de développement du commerce, et UK Export Finance (UKEF), pour couvrir les risques commerciaux et d'investissement des entreprises faisant des affaires en Afrique. La décision actuelle a été soutenue par la Banque européenne d'investissement (BEI) et reflète une tendance qui a vu un nombre record de pays d'Afrique de l'Ouest rejoindre l'agence de garantie multilatérale africaine, le Ghana, le Niger et le Nigéria ayant tous achevé leur adhésion au cours des neuf derniers mois. Cette tendance devrait se poursuivre alors que les pays recherchent un soutien pour garantir les investissements et les flux commerciaux sur le continent afin de gérer les retombées économiques du coronavirus. Sani Yaya, Ministre de l'économie et des finances, a expliqué que l'adhésion à l'ATI était une composante nécessaire de la capacité du Togo à soutenir l'économie et à surmonter la tempête Corona. Reflétant le rôle important joué par ATI dans la croissance économique de la région, la BEI a accordé un prêt concessionnel de 12,5 millions de dollars EU pour couvrir la participation du Togo dans ATI. L'adhésion à l'ATI ouvre la voie aux pays pour réduire leurs niveaux d'endettement, elle fournit également aux investisseurs et aux financiers mondiaux l'assurance que les transactions souveraines et autres investissements sont soutenus par une agence de garantie d'assurance hautement cotée et réputée.

L'inquiétude régionale des paires africaines sur le régime autocratique au Togo a été confirmée par une décision de la Cour de justice de la CEDEAO qui a jugé le 26 juin que la fermeture d'Internet, lors des grandes manifestations de l'opposition au Togo contre la modification de la constitution pour permettre une autre présidence de Faure Gnassingbé en 2017, était illégale. Cela a eu des répercussions sur l'évaluation internationale des fermetures supplémentaires d'Internet pendant les présidentielles de 2020. Peu avant, le 11 juin, la 14e éd. du Global Peace Index 2020, produit par l'Institute for Economics \& Peace (IEP, Sydney, New York), avait révélé que le Togo était en bas de la paix des régions, classant le Togo au 108e rang sur 163 dans le classement international des pays pacifiques en 2020, et 24e en Afrique subsaharienne.

Néanmoins, l'intimidation de l'opposition s'est poursuivie avec tous les moyens. Selon une enquête menée par Le Monde (Paris) et The Guardian (Londres), publiée le 3 août, basée sur les recherches du renommé Citizen Lab (Toronto), le gouvernement de Lomé avait utilisé un logiciel israélien très sophistiqué " Pegasus " pour cibler les religieux catholiques et les militants de la société civile pour espionner les téléphones portables et le trafic Internet de la DMK d'opposition. Officiellement, le logiciel avait été vendu au 
Version française étendue de l'auteur de : Kohnert, D. (2022): Togo. In : Albert K. Awedoba / Benedikt Kamski / Andreas Mehler / David Subudubudu (eds.), Africa Yearbook. Vol. 17 - Politics, economy and society South of the Sahara in 2020. Leiden \& Boston: Brill:, à paraitre

gouvernement de Lomé pour lutter contre le terrorisme et les crimes graves. L'étroite collaboration de longue date d'Israël et du Togo dans la politique africaine, la coopération militaire et l'espionnage, basée sur un bénéfice mutuel depuis l'époque du défunt dictateur Eyadéma, avait déjà suscité dans le passé des protestations des organisations de défense des droits et de l'opposition.

Le 9 septembre, la CEDEAO a annoncé qu'elle retirerait progressivement sa force d'intervention en Guinée-Bissau. Un premier groupe composé de 130 hommes principalement des forces spéciales togolaises arrivés en Guinée-Bissau en novembre 2019 pour renforcer le contingent sous-régional stationné à Bissau depuis 2012, avait déjà quitté Bissau le 27 août. Les autres troupes de la CEDEAO, notamment des Nigérians , Sénégalais et Burkinabé, partiront progressivement d'ici décembre 2020, passant le relais à l'armée de Guinée-Bissau.

Début novembre (4 novembre), Lomé s'apprêtait à ratifier la convention avec le Bénin voisin sur le statut du fleuve Mono, bordant les deux pays, et la création de l'Autorité du bassin du Mono (ABM). L'avant-projet de loi autorisait le Togo à ratifier la convention Mono, qui lierait le Togo et le Bénin sur leur fleuve frontalier, avait été signée déjà le 30 décembre 2014, à Cotonou. Le projet de loi visait à assurer un développement intégré et durable du bassin du Mono en étroite collaboration entre le Bénin et le Togo.

Le 1er décembre, l'Assemblée nationale a adopté un projet de loi autorisant l'État togolais à ratifier l'accord portant création de l'Organisation africaine de la propriété intellectuelle (OAPI), dont le siège est à Yaoundé, qui regroupe 17 États africains majoritairement francophones. La ratification de cet accord adopté en décembre 2015 à Bamako permettra au Togo de mieux protéger les inventions et créations de ses citoyens et de lutter efficacement contre la contrefaçon. L'accord représente également un pas en avant dans le renforcement de la modernisation du secteur privé et de l'arsenal juridique togolais de contrôle des produits pharmaceutiques aux frontières.

L'UE et ses États membres, notamment la France et l'Allemagne, ont continué d'aider le Togo. L'UE a annoncé le 15 janvier son appui budgétaire de plus de 10,5 milliards $\mathrm{F}$ CFA (16 millions d'euros) pour la « consolidation de l'État, phase 3 (CCET 3) » pour l'année 2019. La KfW allemande a fourni 5 millions d'euros le 18 mai à renforcer le processus de décentralisation. Depuis 2012, l'Allemagne a déboursé plus de 30 millions de francs CFA (45,7 millions d'euros) pour soutenir la décentralisation au Togo.

Le 10 septembre 2020, des experts du Service de la prévention du terrorisme de l'ONUDC ont tenu une première réunion consultative avec le Comité interministériel sur la prévention et la lutte contre l'extrémisme violent (CIPLEV) du Togo, dans le cadre du projet du Forum Globale Contre le Terrorisme (GCTF), un projet sur le renforcement des capacités dans la région de l'Afrique de l'Ouest. Les faux médicaments tuent des gens et financent la terreur. Les dirigeants africains espèrent faire quelque chose à ce sujet.

Aussi, la France a étendu en août son soutien au Togo et au Ghana pour la surveillance aérienne anti-jihadiste: Déjà engagé au Bénin, au Burkina Faso, au Mali et en Guinée, Paris étendra son programme de soutien aux forces aériennes ouest-africaines au Togo et au Ghana, menacé par des groupes terroristes du Sahel dans le cadre de son 
Version française étendue de l'auteur de : Kohnert, D. (2022): Togo. In : Albert K. Awedoba / Benedikt Kamski / Andreas Mehler / David Subudubudu (eds.), Africa Yearbook. Vol. 17 - Politics, economy and society South of the Sahara in 2020. Leiden \& Boston: Brill:, à paraitre

programme de développement de l'aviation d'observation légère ouest-africaine au Togo et au Ghana. Dans le cadre du projet de coopération Defence Forces Enabling Development (DEFEND) porté par la Direction de la Coopération Sécurité et Défense (DCSD) du Ministère des Affaires Etrangères, Paris s'était déjà engagé pour fournir des avions ultralégers (ULM) d'observation (CS Grouse) au Bénin et au Burkina Faso. Bamako avait actuellement six exemplaires de Tétras et Conakry trois. Par ailleurs, le 16 novembre, Paris a soutenu la marine togolaise pour le combat de la piraterie dans le golfe de Guinée avec des chasseurs en mer Sillinger du même type déjà destinés aux garde-côtes libyens.

En novembre, Lomé a également participé à des efforts de médiation dans la crise malienne. Lors du coup d'État d'août des forces armées maliennes, le président Ibrahim Boubacar Keïta avait été contraint de démissionner et le gouvernement avait été dissous. Plusieurs pays comme la France, les États-Unis et des représentants de l'UA, de l'UE et du Conseil de sécurité des Nations Unies, ainsi qu'Amnesty International, ont condamné à l'unanimité le coup d'État, appelant les soldats à retourner dans leurs casernes. L'Organisation internationale de la francophonie (OIF) a même suspendu le Mali de l'adhésion. Le 7 septembre, la CEDEAO a donné aux dirigeants militaires maliens la date limite du 15 septembre pour nommer un nouveau président civil et Premier ministre. Lomé, de son côté, est resté ouvert aux négociations et a assumé un rôle de médiation pour sauver la junte. Le 13 novembre, le président de transition à Bamako, Bah N'Daw, s'est rendu à Lomé pour des négociations. Mais dans les coulisses, des contacts s'étaient déjà fait dans le plus grand secret au lendemain du putsch, avec notamment un échange de visites en jets privés et de réunions clandestines.

Ces dernières années, un nombre croissant d'étrangers qui transitaient généralement par le Togo pour aller ailleurs ont été arrêtés pour trafic des médicaments contrefaits. Le 19 janvier, sept dirigeants africains de la République du Congo, de la Gambie, du Ghana, du Niger, du Sénégal, de l'Ouganda et du Togo ont signé un accord pour une législation plus stricte visant à criminaliser la vente de faux médicaments lors du sommet FranceAfrique de deux jours sur les médicaments contrefaits, un commerce meurtrier qui fait des centaines de milliers de vies chaque année en Afrique et finance la criminalité transnationale et le terrorisme.

En novembre 2019, la multinationale nigériane Dangote Industries et le gouvernement togolais avaient convenu de développer et de transformer l'industrie togolaise des phosphates et du ciment. En janvier 2020, le premier contrat d'une usine de broyage de ciment de 2,5 millions de tpa avec VRM de la Cimenterie de Côte Ouest-Africaine (CimCo) a été attribué au Groupe Intercem (Cimfaso \& Cimasso / Burkina Faso et CimIvoire / Côte d'Ivoire) par le Groupe CimMetal. Le démarrage de la production était prévu pour le premier trimestre 2021. Pour son implantation et son expansion au Togo, CimCo bénéficiera d'un prêt de 20 milliards de F CFA de la BOAD. La facilité à long terme a été approuvée le 28 septembre par le conseil d'administration de la Banque. La nouvelle installation, nommée Cimco SA, sera stratégiquement située dans le port de Lomé et deviendra la plus grande usine de broyage du pays en termes de capacité. HeidelbergCement a annoncé l'agrandissement de sa filiale togolaise, Cimtogo, en dépensant plus de 30 millions de dollars dans le processus, ce qui créerait $30 \%$ d'emplois directs en plus, en plus des près de 4.000 emplois directs et indirects déjà assurés au Togo. Heidelberg disposait déjà d'une station de broyage à Kara et d'une 
Version française étendue de l'auteur de : Kohnert, D. (2022): Togo. In : Albert K. Awedoba / Benedikt Kamski / Andreas Mehler / David Subudubudu (eds.), Africa Yearbook. Vol. 17 - Politics, economy and society South of the Sahara in 2020. Leiden \& Boston: Brill:, à paraitre

usine de clinker intégrée à Tabligbo. En janvier, le groupe HeidelbergCement et la Coopération allemande au Togo (GIZ) ont annoncé le cofinancement de la formation de 200 habitants de la zone d'extraction de clinker de Tabligbo (80 km au nord-est de Lomé) sans expérience pour devenir menuisiers et maçons.

Compte tenu de l'énorme dette chinoise du Togo et des relations extérieures, habituellement excellentes, entre Lomé et Pékin, le Togo était également susceptible de profiter des annulations de dette des banques chinoises sur des prêts sans intérêt dus d'ici la fin de l'année pour certains pays africains, comme le prévoit Pékin en septembre. Cependant, le président Xi Jinping, qui a présenté les perspectives d'une annulation de dette lors d'un sommet sur la façon dont la Chine et l'Afrique pourraient lutter ensemble contre la pandémie de Covid-19, n'a pas précisé quels pays africains seraient exonérés ou combien de dette serait effacée carrément.

Par ailleurs, Alassane Ouattara, président de la Côte d'Ivoire, a annoncé en septembre de procéder à la mise en œuvre du projet de monnaie commune ouest-africaine ECO «d'ici trois à cinq ans ». Le sujet controversé devait à l'origine être traité dans le cadre d'un accord successeur, longtemps retardé, l'accord de Cotonou entre l'UE et la communauté d'Afrique, des Caraïbes et du Pacifique (ACP) expirant fin 2020. Les renégociations semblaient approcher de la ligne d'arrivée avec un «99\% chance de succès cette année », selon des sources ACP et UE (sources EURACTIV) le 2 septembre. Cependant, à la veille de la pandémie à la mi-février, l'UE et les équipes de négociation $\mathrm{ACP}$, dirigées par la commissaire européenne aux partenariats internationaux, Jutta Urpilainen et le ministre togolais des Affaires étrangères, Robert Dussey, ont convenu de prolonger l'accord existant jusqu'au 6e sommet UE-Afrique, prévu pour décembre 2020, au cas où le sommet ne serait pas reporté en raison de la Covid pandémie.

L'auto-évaluation du Mécanisme Africain d'Évaluation par les Pairs (MAEP) du

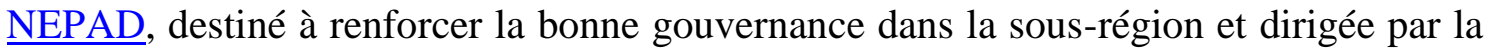
commission nationale Togolaise du MAEP, s'est prolongée. Les premiers efforts pour initier une structure d'évaluation nationale au Togo n'avaient commencé qu'à contrecœur en 2011. Il existe une commission nationale du MAEP, composée de 37 OSC, sélectionnées à la main par le gouvernement, et de membres de l'administration publique. Les membres de la plateforme ont examiné le rapport de l'État du Togo entre mai et août 2018, les résultats étaient toujours en attente.

Le 1er décembre 2020, l'Assemblée nationale a adopté une loi autorisant l'État togolais à ratifier le traité de 2015 pour adhérer à l'Organisation régionale africaine de la propriété intellectuelle (ARIPO). Un mois avant (4 novembre), le Togo avait ratifié une autre convention avec le Bénin voisin sur le statut du Mono-fleuve et la création de l'Autorité du Mono-Bassin qui avait déjà été signée le 30 décembre 2014 à Cotonou.

\section{Développement socio-économique}

En avril, la Banque mondiale et le $\underline{\text { FMI }}$ ont envisagé un soutien supplémentaire considérable au Togo pour contrer l'impact économique de la crise de Corona. Le 29 
Version française étendue de l'auteur de : Kohnert, D. (2022): Togo. In : Albert K. Awedoba / Benedikt Kamski / Andreas Mehler / David Subudubudu (eds.), Africa Yearbook. Vol. 17 - Politics, economy and society South of the Sahara in 2020. Leiden \& Boston: Brill:, à paraitre

avril, la Banque mondiale a approuvé un financement de 8,1 millions de dollars de l'Association internationale de développement (IDA) pour aider le Togo à lutter contre Covid-19 et à mieux répondre aux urgences de santé publique. Un jour auparavant, la Banque mondiale avait déjà approuvé 273 millions de dollars de financement de l'Association internationale de développement (IDA) pour le Togo, le Bénin, le Burkina Faso et le Niger pour faciliter l'accès aux services des personnes vulnérables (en particulier les femmes et les couches les plus pauvres de la société), à travers le programme d'identification unique de l'Afrique de l'Ouest pour l'intégration et l'inclusion régionales (WURI). Et le 3 avril, le FMI avait déjà autorisé un décaissement immédiat de 131,1 M \$ au Togo en lien avec l'achèvement de la 6ème et dernière revue de la performance économique du pays dans le cadre du programme soutenu par l'accord de la Facilité Elargie de Crédit (FEC). Le décaissement a été quatre fois supérieur à ce qui avait été initialement prévu (35 millions de dollars) en raison des implications humaines et économiques de Covid-19. Après trois ans de mise en œuvre du programme appuyé par le FMI, le FMI a estimé que la performance du gouvernement était satisfaisante dans la plupart des secteurs. Ce n'est que dans le secteur financier que les réformes ont connu des retards.

Avant la pandémie de Covid-19, les perspectives économiques de l'économie togolaise étaient encourageantes. La croissance devant atteindre 5,3\% en 2020 et 5,5\% en 2021, grâce aux bonnes performances de l'agriculture et à une saine gestion monétaire. Cependant, sous le choc COVID-19, le Togo était susceptible d'enregistrer une perte de croissance comprise entre $4,6 \%$ et $6,8 \%$ points en 2020 . Au moins, cela éviterait probablement une récession. Le PIB réel en 2020 ne croîtrait que de $0,9 \%$ si la pandémie s'atténuait en juillet (référence), et se contracterait de $1,3 \%$ si elle se poursuivait jusqu'en décembre (pire des cas). Dans le pire des cas, le déficit budgétaire, initialement prévu à $1,5 \%$ du PIB en 2020, s'élargirait jusqu'à $6,4 \%$ en raison de l'augmentation des dépenses de santé et de la baisse des recettes fiscales provoquée par la baisse du niveau général activité économique.

La production de céréales pour le marché intérieur était satisfaisante. Cependant, des problèmes de débouchés pourraient décourager les agriculteurs, selon un communiqué d'Ouro-Koura Agadazi, directeur général de l'Agence nationale de sécurité alimentaire (ANSAT) le 3 mai. En moyenne 70.000 tonnes des surplus de céréales ont été enregistrés sur la campagne agricole 2019-2020, cependant, plusieurs zones de production ont affiché des quantités de céréales invendues, comme la région Nord-Est de Sokodé (plus de 4.200 tonnes) et Elavagnon dans le Mono Est (plus de 5.000 tonnes de maïs). Les préfectures de Bassar, Dankpen, Mo, Sotouboua, Blitta, Tchamba, Doufelgou, Binah, Keran, South Oti, Kpendjal, Tandjoare, Tone ont également été touchées. Agadazi a averti qu'il y avait un risque que les agriculteurs soient démotivés s'il n'y avait pas de mesures pour faciliter la commercialisation de leurs produits, ce qui pourrait avoir des implications néfastes pour la sécurité alimentaire. Pour assouplir l'attitude commerciale spéculative de certains commerçants en raison de la crise Corona, l'ANSAT a ouvert ses reserves, et en cinq semaines, 91,5 tonnes de céréales ont été mises sur le marché local sur plus de 13.000 tonnes de stock de sécurité nationale. Ainsi, le prix de $2,5 \mathrm{~kg}$ de maïs, qui était passé de $400 \mathrm{~F} \mathrm{CFA}$ à 700 voire 800 , est retombé au prix normal. L'ANSAT a annoncé d'augmenter le stock de sécurité de 13.000 à 15.000 tonnes et d'aider les agriculteurs à exporter leurs excédents sur 
Version française étendue de l'auteur de : Kohnert, D. (2022): Togo. In : Albert K. Awedoba / Benedikt Kamski / Andreas Mehler / David Subudubudu (eds.), Africa Yearbook. Vol. 17 - Politics, economy and society South of the Sahara in 2020. Leiden \& Boston: Brill:, à paraitre

demande, comme il l'avait déjà fait ces dix dernières années, vers les pays de la sousrégion, et même vers l'Europe, l'Asie et les États-Unis.

Le déficit de la balance courante devrait suivre une trajectoire similaire que les prevision pour le PIB. Prévu à 3,2\% du PIB, il devrait s'aggraver à 5,7\% ou peut-être $7 \%$ du PIB en 2020 sous l'effet de la baisse des exportations, de la baisse des envois de fonds des migrants et des IDE dans les principaux secteurs économiques. Les principales exportations du Togo, notamment les réexportations, le coton, les phosphates, le café et le cacao, seraient également affectées négativement. Les taux élevés de sous-emploi en général (estimés de $28 \%$ à 33\%), notamment parmi les récents bacheliers $(65 \%)$, sont restés alarmants.

Dans l'ensemble, l'économie et le budget du gouvernement restent volatils et sujets aux chocs extérieurs. Le 18 décembre 2020, le Parlement a voté la loi de finances pour 2021. Selon les prévisions, le budget 2021 sera équilibré en ressources avec des dépenses à 1 521,6 milliards F CFA, soit 2,84 milliards \$. Les recettes budgétaires s'élèvent à 839,6 milliards de F CFA (1,57 milliards de dollars), inférieures aux dépenses budgétaires de 1120,4 milliards de francs CFA (2,09 milliards de dollars), soit un déficit budgétaire de 280,8 milliards de francs CFA (524,78 millions de dollars). Le déficit représente $6,1 \%$ du PIB et serait « entièrement financé par le solde excédentaire des opérations de trésorerie », selon le ministre des Finances, Sani Yaya. Le budget consacre 445,1 milliards de F CFA (831,83 millions de dollars) aux secteurs sociaux, en hausse de 10,8\% par rapport à 401,7 milliards de francs CFA (750,73 millions de dollars) en 2020. 65,9 milliards de francs CFA (123,16 millions de dollars), soit 8,2\% du budget, sont alloués au secteur de l'eau, pour atteindre un taux de service moyen d'au moins $80 \%$ en milieu urbain et rural. L'agriculture bénéficiera de 65,7 milliards de $\mathrm{F}$ CFA (122,78 millions de dollars), tandis que 77,9 milliards de francs CFA (145,58 millions de dollars) seront réservés au secteur de la santé, soit 10\%, et l'éducation est allouée 195,5 milliards de francs CFA (365,36 millions de dollars), soit 25,1\% de l'allocation du budget 2021.

La privatisation des deux banques publiques restantes, la Banque du commerce et de l'industrie (BTCI) et l'Union des banques togolaises (UTB), à laquelle le gouvernement s'oppose depuis des années, traîne encore. En octobre, cinq candidats potentiels avaient été sélectionnés pour le rachat des banques. La sélection se fera en un seul lot. Ralenti par la pandémie de Covid-19, le processus de vente des deux banques publiques avait été relancé par la Commission de privatisation dirigée par le ministre des Finances, Sani Yaya, avec l'ouverture de la « data room ». Il appartenait aux candidats présélectionnés de faire leurs offres maintenant.

Par le passé, le gouvernement a mené une politique monétaire et budgétaire axée sur la stabilité. Selon le FMI, le Togo a respecté le critère de convergence de l'UEMOA d'un déficit budgétaire ne dépassant pas 3\% du PIB. Pour 2020, le déficit budgétaire et le déficit de financement de la balance des paiements devraient se creuser en raison des dépenses de santé supplémentaires et d'autres impacts du COVID-19 sur l'économie. Cependant, l'assainissement budgétaire et la réduction de la dette publique se sont poursuivis en 2019. Des réformes sont en cours pour stimuler la mobilisation des ressources nationales, renforcer le système de GFP et améliorer l'environnement des affaires. Les projections de croissance pour 2020 ont été abaissées de 5,5\% à 3\%. Le 
Version française étendue de l'auteur de : Kohnert, D. (2022): Togo. In : Albert K. Awedoba / Benedikt Kamski / Andreas Mehler / David Subudubudu (eds.), Africa Yearbook. Vol. 17 - Politics, economy and society South of the Sahara in 2020. Leiden \& Boston: Brill:, à paraitre

déficit budgétaire devrait se creuser, passant d'une projection initiale de $1,9 \%$ du PIB à $3,6 \%$, en raison de l'augmentation des dépenses de santé et de la perte de revenus. La balance des paiements a montré un déficit de financement de $1,7 \%$ du PIB. Le gouvernement a demandé en 2020 une augmentation de l'accès de 48,7\% du quota (71,49 millions de DTS) pour répondre au besoin de financement urgent découlant de son plan de contrôle de la propagation du COVID-19 et d'atténuation de ses implications économiques.

Le système de protection sociale du Togo est sous-développé. Il n'est disponible que pour les employés du gouvernement et ceux employés dans le secteur formel. Le 9 octobre 2020, le gouvernement a annoncé que le salaire minimum togolais (SMIG garanti) pourrait prochainement être revu à la hausse. En vigueur depuis 2012, le SMIC, qui oscille autour de 35.000 à $38.000 \mathrm{~F}$ CFA $(64,03 €)$, n'est pas toujours appliqué partout. Les travailleurs se plaignaient régulièrement de conditions de travail précaire caractérisées par de mauvaises conditions d'hygiène et de sécurité et le non-paiement du salaire minimum.

La pandémie COVID-19 a également eu un effet perceptible sur le niveau d'exclusion sociale, notamment des personnes employées dans le secteur informel. La pandémie pourrait anéantir les quatre cinquièmes de la croissance projetée du Togo en 2020 selon un rapport de la BM. Environ 62\% des emplois sont à risque, $49 \%$ dans le secteur des services et $13 \%$ dans le secteur industriel. Les petites et moyennes entreprises ont été particulièrement touchées. Les taux de pauvreté, les inégalités et le développement humain seront très probablement encore aggravés. Pour contrer les effets négatifs sur les pauvres et les vulnérables, la Financière Africaine de Micro-Projets (FINAM), première société anonyme de microfinance, s'est lancée dans la digitalisation de ses services en créant « Finam Mobile » en réponse à la crise Covid. L'application permet de gérer son compte à la maison, y compris des dépôts et des retraits à partir du 11 septembre 2020.

Selon les données officielles présentées par le gouvernement en 2020, le taux de chômage (officiel) se situait entre 1,7\%. et 1,9\% au cours des dix dernières années (2010-2020). Celui des jeunes chômeurs (total, 15 à 24 ans) à environ 3,4\% en 2020 avant l'impact économique de la pandémie Corona. À titre de comparaison, la moyenne mondiale en 2020 basée sur 182 pays avant Corona était de 15,90\% (WB; Global Economy.com). Cependant, le taux de sous-emploi est passé de 22,8\% (2011) à 24,9\% (2015; INSEED; aucune mise à jour disponible à partir de Décembre 2020), soit un total d'au moins 28,3\% de chômeurs et de sous-employés, majoritairement les jeunes qui représentaient environ $35 \%$ de la population. Le gouvernement a tenté de contrer cette tendance avec un succès limité par son Agence Nationale de Volontariat au Togo, (ANVT). Depuis 2011, plus de 43.000 candidats ont été enregistrés. Au total, 8.989 personnes, soit 4.610 femmes et 4.348 hommes, dont 31 personnes handicapées, ont été touchées en 2020.

Le 11 septembre, la Financière Africaine de Micro-Projets (FINAM), s'est lancée dans la digitalisation de ses services en créant « Finam Mobile » en réponse à la crise Covid. L'application vous permet de gérer votre compte à la maison, y compris d'effectuer des dépôts et des retraits. La FINAM était la première société anonyme de microfinance, fondée en 2015. Elle avait lancé la première édition de sa campagne de 1.000 microprojets pour lutter contre le chômage des jeunes. Celles-ci peuvent bénéficier, sans 
Version française étendue de l'auteur de : Kohnert, D. (2022): Togo. In : Albert K. Awedoba / Benedikt Kamski / Andreas Mehler / David Subudubudu (eds.), Africa Yearbook. Vol. 17 - Politics, economy and society South of the Sahara in 2020. Leiden \& Boston: Brill:, à paraitre

dépôt préalable, mais avec de simples garanties des membres de la FINAM, des prêts jusqu'à $600.000 \mathrm{~F}$ CFA pour la réalisation de leurs micro-projets.

La crise du coronavirus a accéléré la mise en place d'un revenu de solidarité universel, appelé «Novissi » (signifiant «fraternité » et «vivre ensemble » en langue Ewe) par le Fonds national de solidarité et économique en avril. Il était versé deux fois par mois et principalement destiné aux personnes vivant du salaire journalier, sans sécurité sociale et affectées par une baisse d'activité, comme l'explique Cina Lawson, la ministre de l'Economie numérique. Novissi était un appareil $100 \%$ numérique qui a touché près de 570.000 pauvres méritants entre le 8 avril et le 6 juin 2020, date de la levée des couvrefeux au Grand Lomé et à la préfecture de Tchaoudjo en Région Centrale. Le programme a été étendu au canton rural de Soudou dans la région de Kara au nord-est du Togo lorsque la circulation du coronavirus a entraîné une réduction drastique des activités. Pour soutenir les individus et les ménages qui avaient été touchés par la pandémie COVID-19, un programme de transfert monétaire mobile pour les travailleurs du secteur informel a été mis en place. En composant un numéro de mobile à trois chiffres, par ex. vendeurs ambulants, cuisiniers en bord de route et autres personnes du secteur informel dont les activités ont disparu au plus fort de la pandémie Covid-19 ont pu générer un portefeuille électronique de bénéficiaires via leur smartphone et ainsi créer un revenu minimum pour garantir leur survie. Les femmes percevaient une allocation mensuelle plus élevée (12.250 F CFA ou 18,60 €) que les hommes (10.500 F CFA ou $16 €$ ). Au total, 11,3 Mrd. Des F CFA (17,2 M $€$ ) ont été distribués, financés par le Fonds National de Solidarité et de Relance Economique du Togo et par des partenaires d'aide tels que l'Agence Française de Développement (AFD) et des dons privés. Les bénéficiaires devaient recevoir directement, par inscription mobile, une subvention de l'État d'au moins $30 \%$ du salaire minimum, avec des versements allant de 10.500 millions de F CFA (18 millions USD) à 20000 millions FCFA (34 millions USD). Au 5 mai, 1,3 million de personnes s'étaient inscrites, dont 500.000 ont reçu un paiement Novissi. Sur la base des données du programme, $65 \%$ des bénéficiaires étaient des femmes. Le programme de transferts monétaires devait durer 3 mois pour un coût de 36 milliards de F CFA (61 millions USD; 1,1\% du PIB). Le programme de Novissi a été révisé à la fin du mois de juin, l'éligibilité étant limitée aux travailleurs des districts spécifiques enregistrant un taux de contagion élevé. Au total, 1,4 million de personnes s'étaient inscrites et près de 600.000 ont reçu un paiement Novissi pour un coût total de 11,4 milliards de F CFA (19 millions USD; 0,3\% du PIB) jusqu'à présent. Le gouvernement entendait continuer sur cette voie, en s'appuyant sur sa couverture mobile pour avancer et faire en sorte que chaque citoyen dispose de trois choses essentielles : une identité biométrique numérique, un téléphone portable et un compte bancaire mobile. Cette mesure d'urgence innovante a été la première à être utilisée à cette échelle en ASS et pourrait inspirer d'autres programmes de revenu universel sur le continent. Le gouvernement entendait continuer sur cette voie, s'appuyant sur sa couverture mobile pour avancer et voulait faire en sorte que chaque citoyen dispose de trois choses essentielles : une identité biométrique numérique, un téléphone portable et un compte bancaire mobile. Des programmes similaires ont également été testés en Afrique du Sud et au Kenya.

Le 24 octobre, le Togo est entré pour la première fois sur le marché des titres publics de l'Union monétaire ouest-africaine (UMOA), pour la première sortie au cours du dernier semestre 2020. Le pays a collecté 27,5 milliards de francs CFA auprès d'investisseurs, 
Version française étendue de l'auteur de : Kohnert, D. (2022): Togo. In : Albert K. Awedoba / Benedikt Kamski / Andreas Mehler / David Subudubudu (eds.), Africa Yearbook. Vol. 17 - Politics, economy and society South of the Sahara in 2020. Leiden \& Boston: Brill:, à paraitre

selon l'Agence UMOA-Titres. L'opération a consisté en une émission simultanée d'Obligations du Trésor Assimilables (OAT) d'une maturité de 5 et 7 ans, levées 27,5 milliards F CFA. Aux taux d'intérêt annuels respectifs de 6,4\% et 6,5\%, l'OAT à 5 ans a collecté 3,3 milliards de francs CFA, la dernière maturité de 7 ans ayant conservé 24,2 milliards de francs CFA. Pour le 4ème trimestre, le Togo prévoit de mobiliser 60 milliards de francs CFA auprès des investisseurs de la zone UMOA. Le pays a déjà collecté 573,43 milliards de francs CFA sur le marché financier régional pour les trois premiers trimestres de l'année.

La production cotonnière de la campagne 2019-2020 a donné un rendement médiocre de 116.000 tonnes, contre 150.000 tonnes prévues, selon la Nouvelle Société Cotonnière du Togo (NSCT). Une augmentation de la superficie des terres cultivées déclenchée par les efforts du gouvernement pour accroître l'efficacité dans ce secteur par le service de vulgarisation propageant les meilleures pratiques et une meilleure coordination des producteurs est censée entraîner une croissance de la production de coton, bien que les prix du coton probablement plus bas pour la saison 2020-21 qu'en 2019. La nouvelle campagne coton 2020-2021 a été officiellement lancée à Kara le 19 mai. Le prix du coton graine a été fixé à $225 \mathrm{~F} / \mathrm{kg}$ pour le premier choix.

En avril, le Togo a signalé l'épidémie de peste porcine africaine dans une ferme au nordouest de Lomé, où 44 porcs sont morts de la maladie depuis le 18 avril. Le gouvernement a pris une série de mesures de prévention et de désinfection pour enrayer la propagation de la maladie, y compris l'abattage de tous les porcs de la ferme.

En août, le Togo a annoncé l'éradication d'un fléau, la trypanosomiase africaine, mieux connue sous le nom de «maladie du sommeil », un problème de santé publique et une maladie endémique dans 36 pays d'Afrique subsaharienne où l'on trouve la mouche tsétsé. Il affecte à la fois les humains et les animaux, en particulier le bétail. Grâce aux actions du gouvernement togolais, des communautés locales et de l'Organisation mondiale de la santé (OMS), la maladie a été éradiquée. Les contrôles soutenus ont réduit le nombre de cas de 95\%, passant de 10.000 en 2009 à 977 en 2018. Cependant, selon les estimations, 300.000 cas en ASS n'ont pas été diagnostiqués et donc non traités. La population à risque estimée était de 65 millions de personnes. Le Togo n'avait signalé aucun nouveau cas depuis plus d'une décennie. L'épidémie la plus récente en ASS a commencé en 1970 et a duré jusqu'à la fin des années 1990. La trypanosomiase ne posait pas de problème de santé publique à l'horizon 2020, et l'interruption de la transmission (zéro cas) était envisagée pour 2030.

Les dommages environnementaux ont également eu des effets négatifs graves sur l'économie, par exemple concernant l'érosion côtière. En juin, le village de Doevikope sur la plage de Baguida, à l'est de Lomé, les trois quarts des habitants se sont éloignés depuis que l'océan a englouti de précieuses terres agricoles, le terrain de jeu de l'école et le cimetière. L'érosion côtière qui a affecté le littoral océanique et ses lacs adjacents au Lac du Togo et au lac Boko au cours de la période 1988-2018, a entraîné un élargissement du Lac du Togo en moyenne de $1,55 \mathrm{~m} / \mathrm{an}$, tandis que le littoral du lac Boko s'est rétréci de $1,25 \mathrm{~m} /$ an. Pour le littoral de l'océan, la régression a varié de 1,66 à $5,25 \mathrm{~m} /$ an. La plage barrière a connu une immersion moyenne de 9,25 ha / an. Les prévisions basées sur le taux moyen d'immersion de la plage barrière de 9,25 ha / an ont montré que cette dernière est exposée à un danger d'immersion continue qui toucherait 
Version française étendue de l'auteur de : Kohnert, D. (2022): Togo. In : Albert K. Awedoba / Benedikt Kamski / Andreas Mehler / David Subudubudu (eds.), Africa Yearbook. Vol. 17 - Politics, economy and society South of the Sahara in 2020. Leiden \& Boston: Brill:, à paraitre

plus de $7 \%$ de sa superficie actuelle (6557,33 ha) d'ici 2070. Le bord de l'eau de la capitale Lomé perd environ 10 mètres par an à cause de l'érosion causée par l'homme, et de petits villages situés le long de la côte comme Baguida, Gbodjomé, Agborafo se plongent successivement dans la mer.

En ce qui concerne la déforestation, le Togo s'est classé dans la fourchette inférieure (165e rang sur 190 pays). De 2001 à 2019, le Togo a perdu 55,9 kha de couvert arboré, soit une diminution de $10 \%$ du couvert arboré depuis 2000 , et 11,3 Mt d'émissions de $\mathrm{CO}_{2}$. De 2002 à 2019, le pays a perdu 299 ha de forêt primaire humide, soit $0,56 \%$ de la perte totale de son couvert arboré au cours de la même période. La superficie totale de forêt primaire humide au Togo a diminué de $82 \%$ pendant cette période (Global Forest Watch, 2020) . Les principales régions des régions du Plateau et du Centre étaient responsables de 58\% de la perte totale du couvert arboré entre 2001 et 2019. Cette région a enregistré la plus grande perte de couvert arboré avec 32,6 kha contre une moyenne de 11,2 kha. la région ouest des bois entre Atakpamé et Sokodé (carte interactive, Global Forest Watch, 2020).

Selon les directives de l'OMS, la qualité de l'air au Togo est considérée comme dangereuse. Les données les plus récentes (avril 2020) indiquent que la concentration moyenne annuelle de PM2,5 dans le pays est de $36 \mu \mathrm{g} / \mathrm{m} 3$, dépassant le maximum recommandé de $10 \mu \mathrm{g} / \mathrm{m} 3$. Les facteurs contribuant à la mauvaise qualité de l'air au Togo sont les industries minières et du ciment, les émissions des véhicules et la combustion des déchets. Le gouvernement a noté une croissance exponentielle des voitures et des motos. En février, la CEDEAO, y compris le Togo, a accepté d'adopter une norme régionale sur l'essence et les carburants diesel importés de 50 parties par million (ppm) à partir de janvier 2021, les raffineries locales étant données jusqu'en janvier 2025 pour s'y conformer. Quatre ans auparavant, en décembre 2016, le Togo et quatre pays voisins (Nigéria, Ghana, Bénin, Côte d'Ivoire) avaient déjà convenu en principe d'interdire les importations de combustibles sales en provenance d'Europe. L'OMS a classé ces risques pour la santé parmi les principaux risques sanitaires mondiaux, associés aux maladies cardiaques, au cancer du poumon et aux problèmes respiratoires. En septembre, un nouveau rapport du groupe international de surveillance des ressources Stakeholder Democracy Network (SDN) partiellement financé par le fonds anti-corruption, stabilité et sécurité du ministère britannique des Affaires étrangères, a révélé que même le carburant du marché noir fabriqué à partir de pétrole volé par les «raffineries de brousse », cachées profondément dans les ruisseaux et les marais du delta du Niger, étaient moins polluantes que le diesel et l'essence hautement toxiques que l'Europe exporte vers l'Afrique. Shell, Exxon, Chevron et d'autres grandes compagnies pétrolières extraient et exportent jusqu'à $2 \mathrm{~m}$ de barils par jour de brut «Bonny Light» de haute qualité et à faible teneur en soufre du delta du Niger. Mais seules de petites quantités de ce pétrole sont raffinées dans le propre pays parce que ses quatre raffineries publiques sont dysfonctionnelles ou ont fermé. Au lieu de cela, les concessionnaires internationaux exportent vers le Nigéria environ 900.000 tonnes par an de carburant de qualité inférieure «sale ", fabriqué dans des raffineries néerlandaises, belges et dans d'autres raffineries européennes. Environ $80 \%$ des produits pétroliers du Nigéria proviennent des Pays-Bas et de la Belgique. Le diesel importé «non officiel» moyen testé a dépassé le niveau des normes de soufre de l'UE 152 fois, et 40 fois le niveau de l'essence. En conséquence, le Nigéria se classe au quatrième rang mondial 
Version française étendue de l'auteur de : Kohnert, D. (2022): Togo. In : Albert K. Awedoba / Benedikt Kamski / Andreas Mehler / David Subudubudu (eds.), Africa Yearbook. Vol. 17 - Politics, economy and society South of the Sahara in 2020. Leiden \& Boston: Brill:, à paraitre

pour les décès dus à la pollution atmosphérique. On estime que 114.000 personnes meurent prématurément de la pollution atmosphérique chaque année.

Lomé a tenté de lutter contre la pollution atmosphérique par son Plan national de réduction de la pollution atmosphérique et des polluants climatiques de courte durée, adopté et publié par le ministre de l'Environnement en 2020. Il comprenait des mesures prioritaires et des actions qui réduiraient considérablement les SLCPs. Ainsi, elle récolterait les multiples avantages de l'amélioration de la qualité de l'air, de la lutte contre le changement climatique et de la réalisation d'avantages connexes comme l'amélioration de la santé et de la productivité agricole. Une mise en œuvre complète entraînerait une réduction de $67 \%$ du carbone noir, de $70 \%$ des particules fines et de $56 \%$ du méthane d'ici 2040.

En février, trois nouveaux acteurs ont rejoint le projet «Cizo » un projet qui avait déjà rencontré avec succès le défi d'électrifier 40.000 ménages dans le Bénin voisin en 2019. Il s'agit de fournisseurs hors réseau, à savoir la société ougandaise Fenix International, Solergie et Moon, filiale du géant Français de l'énergie Engie. Fenix sera en concurrence avec Solergie, qui opère au Togo dans le cadre d'un partenariat avec le géant pétrolier français Total. Une société belge propose une solution appelée « SolergieBox ». Il s'agit d'un mini-réseau composé de panneaux solaires, d'un onduleur et d'une batterie pour stocker l'électricité. Ce système solaire hors réseau peut alimenter 8 foyers en milieu rural. Les ménages connectés au mini-réseau peuvent payer leurs factures via «mobile money », un système de paiement par téléphone mobile. Le téléphone mobile est également un équipement clé du service proposé par Moon. Cette société française, également impliquée dans le projet «Cizo », propose des kits solaires domestiques. Le sien est composé de panneaux solaires, d'un système de stockage et de ports USB pour recharger les téléphones portables. Contrairement à d'autres fournisseurs de kits solaires, sa solution est accompagnée d'un smartphone (Moonphone) sur lequel est installée une application qui permet le paiement du kit solaire par petites sommes (en pay-as-you-go).

Selon le ministère en charge de l'élevage, la campagne de transhumance 2019/2020 qui a pris fin le 31 mai, s'est déroulée sans incident majeur. Au total, quelque 30.000 têtes de bétail ont circulé sur le territoire pendant cette période. De forts mouvements transfrontaliers de bétail saisonniers provenant principalement du Burkina Faso et du Niger, mais aussi entre le Togo et le Nigéria ont été signalés au cours de la période d'enquête. Des liens commerciaux transfrontaliers solides pour le bétail ont été signalés par l'OCDE en 2020.

Le 10 septembre 2020, des experts du Service de la prévention du terrorisme de l'ONUDC ont tenu une première réunion consultative avec le Comité interministériel sur la prévention et la lutte contre l'extrémisme violent (CIPLEV) du Togo, dans le cadre du projet du Forum mondial contre le terrorisme (GCTF), un projet sur le renforcement des capacités dans la région ouest-africaine. Les faux médicaments tuent des gens et financent la terreur. Les dirigeants africains espèrent faire quelque chose à ce sujet. Le 18 janvier 2020, il a été signalé que de fausses pilules avaient été importées au Togo, venant subrepticement de Chine, d'Inde et du Nigéria. Ils étaient emballés comme des remèdes contre la fièvre et les éruptions cutanées et ont été distribués au coin des rues, par exemple à Lomé, promettant de soulager la souffrance à une fraction 
Version française étendue de l'auteur de : Kohnert, D. (2022): Togo. In : Albert K. Awedoba / Benedikt Kamski / Andreas Mehler / David Subudubudu (eds.), Africa Yearbook. Vol. 17 - Politics, economy and society South of the Sahara in 2020. Leiden \& Boston: Brill:, à paraitre

du coût. Mais les faux médicaments tuent des dizaines de milliers de personnes chaque année dans un commerce mondial de contrefaçon d'une valeur estimée à 200 milliards de dollars, contrecarrant les progrès dans la lutte contre le paludisme et d'autres maladies potentiellement mortelles, disent les experts, tout en finançant le crime organisé.

Le Togo reste également un refuge pour le blanchiment d'argent. Le 20 septembre, CENOZO, une unité de rapport d'enquête en Afrique de l'Ouest, a révélé que «Kassoum Zombre, Sarl $U$ », une société togolaise de Lomé, avait été identifiée dans une transaction suspecte avec Saber Pte Ltd, une société de Singapour, connu comme un paradis fiscal pour les géants asiatiques, qui avait son siège social local à Atakpamé. L'affaire serait liée à une transaction financière illégale via l'Iran pour financer des réseaux terroristes dans la zone frontalière au nord du Togo, où la menace djihadiste s'était accrue ces dernières années à la frontière avec le Burkina-Faso. Cinkassé, à la frontière avec le Burkina Faso et le Ghana, à environ $38 \mathrm{~km}$ de Dapaong, était réputé comme le Dubaï de la sous-région, un épicentre de riches commerçants (moguls) et une plaque tournante pour la contrebande et les activités obscures, par ex. des combattants du redoutable groupe Ansaroul, dégradant la situation sécuritaire. Même si le Togo avait été jusqu'ici apparemment épargné de véritables actes terroristes, le risque d'infiltration restait évident. Dapaong, Cinkassé, Yemboate et d'autres localités de la région de Savannah au nord du Togo limitrophe du Burkina Faso ont été classées «Zone Rouge » où la présence de jihadistes avait été remarquée par la population. Le 15 février 2019, le père César Fernández, l'un des premiers prêtres salésiens espagnols en mission au Togo depuis 1982, avait été tué dans une attaque terroriste au poste de contrôle douanier de Nouhao, situé entre Cinkasse (Togo) et Bitou (Burkina Faso).

En janvier 2020, 1,71 millions de Togolais étaient connectés à Internet selon une étude publiée par «Hootsuite and We Are Social» sur l'utilisation du Web et des réseaux sociaux au Togo. Une progression de 7,8\% par rapport à 2019. Le taux global de pénétration d'Internet est désormais de $21 \%$, dont $43,1 \%$ via mobile, $56,1 \%$ par ordinateur, 0,7\% par tablette. 650.000 abonnés étaient actifs sur les réseaux sociaux (+ $14 \%$ entre avril 2019 et janvier 2020). 580.000 utilisateurs accèdent régulièrement à Facebook (95,9\% par mobile), 72.000 à Instagram, 170.000 à Linkedin. Il y avait 3 macro-influenceurs Instagram au Togo avec une moyenne de 81.860 followers. La portée totale de tous les macro-influenceurs Instagram togolais combinés est de 245.581 followers. TikTok serait également de plus en plus utilisé par les adolescents togolais. Des influenceurs comme le comédien influent \#Roland_Tikena compteraient plus de 12 millions vues. La ministre togolaise des Postes, de l'Économie numérique et des Innovations technologiques, Cina Lawson, a annoncé le 28 février 2020 que d'ici 2022, $90 \%$ de la population aura accès aux services Internet haut débit fixes et mobiles. La pénétration réelle du haut débit fixe / mobile était estimée à 35\% à fin 2019.

Le 21 octobre, le nouvel archevêque de Lomé Yves Nicodème Anani Barrigah-Benissan (nommé en 2019) a été annoncé lauréat du Grand Prix de littérature togolaise 2020. Il avait reçu le prix grâce à son œuvre intitulée «Le trône royal ». Il s'agit d'une pièce publiée par Nouvelles Editions Africaines du Togo (NEA-Togo) en 1993. Il a également publié plusieurs autres livres et a été auteur et compositeur de plusieurs chansons. 
Version française étendue de l'auteur de : Kohnert, D. (2022): Togo. In : Albert K. Awedoba / Benedikt Kamski / Andreas Mehler / David Subudubudu (eds.), Africa Yearbook. Vol. 17 - Politics, economy and society South of the Sahara in 2020. Leiden \& Boston: Brill:, à paraitre

La tendance de l'IDH au Togo a progressé au cours des trois dernières décennies, passant de 0,4 en 1990 à 0,51 pour 2018 (rapport IDH, 2019, le rapport le plus récent en 2020), soit une augmentation de $26,6 \%$, ce qui place le pays dans la catégorie de faible développement humain, le positionnant à 167 pays sur 189. Cependant, lorsque la valeur est corrigée de l'inégalité, l'IDH tombe à 0,350 , une perte de $31,7 \%$ due à l'inégalité dans la distribution des indices de dimension de l'IDH. La valeur de l'IDH féminin 2018 pour le Togo est de 0,459 , contrairement à 0,561 pour les hommes, ce qui donne une valeur de l'indice de développement du genre (IDG) de 0,818. La tendance générale a également été reflétée par le Rapport sur le bonheur dans le monde de l'ONU de 2020 où le Togo se classait toujours en bas (135) des 153 États évalués pour la période 2017 à 2019, même s'il comptait, aux côtés du Bénin voisin, parmi les meilleurs bonheurs de 2008-2012 à 2017-2019.

Dans l'indice de la liberté du commerce et des affaires de la Banque mondiale 2020, basé sur la réglementation des affaires dans 190 économies, les meilleurs améliorateurs étaient le Togo (rang 97 sur 190 (Somalie en bas)), aux côtés de l'Arabie saoudite, du Jordon, de Bahreïn, du Tadjikistan, du Pakistan, du Koweït, de la Chine, de l'Inde et du Nigéria. Cependant, l'indice WB Doing Business a fait l'objet de critiques sévères en 2020 en raison de son prétendu biais politique et méthodologique (libre-échange). Plus les réglementations sont réduites, meilleur est le classement d'un pays. Par conséquent, le 27 août 2020, la Banque mondiale a annoncé qu'elle suspendrait le rapport Doing Business pour irrégularités dans les données jusqu'à ce qu'elle procède à un examen et à un audit. L'arrêt du rapport a été salué par les syndicats, les universitaires et les groupes de défense des droits de l'homme. En fait, le rapport Doing Business a sapé le progrès social et favorisé les inégalités. Depuis son lancement en 2003, il a suscité l'indignation pour son parti pris anti-réglementation. Inspiré de 1 ' »Index of Economic Freedom » de la conservatrice Heritage Foundation, le rapport a encouragé les pays à participer à «l'expérience de déréglementation », notamment des réductions de la protection de l'emploi, des cotisations de sécurité sociale plus faibles (dénommées «taxe sur le travail ») et une moindre fiscalité des entreprises. Ainsi, un pays se classe mieux lorsque ses cotisations de sécurité sociale sont faibles, c'est-à-dire lorsque les salariés perçoivent des prestations de protection sociale moindres pour leur famille et prennent leur retraite avec de faibles pensions. Les pays obtiennent également de meilleurs classements si l'impôt sur les sociétés est faible, peu importe si cela va générer davantage d'inégalités et priver de ressources pour le développement national durable. Jusqu'à présent, le manque de transparence et de prévisibilité et les coûts de transaction informels élevés empêchent un IED robuste. Le taux tarifaire moyen appliqué est de 11,4\%. Cependant, de multiples barrières non-tarifaires au commerce entravent le commerce extérieur. Néanmoins, l'indice 2020 de la liberté du commerce et des affaires s'est amélioré, principalement en raison des améliorations apportées au démarrage d'une entreprise, au traitement des permis de construire, à l'obtention d'électricité, à l'enregistrement de la propriété et à l'obtention de crédit.

La réforme du code des investissements s'inspire des progrès du Rwanda au cours des 10 dernières années à cet égard. Plusieurs délégations togolaises se sont rendues à Kigali pour en savoir plus sur les réformes réussies. Le président Faure Gnassingbé avait apparemment la vision d'être numéro un en Afrique de l'Ouest dans Doing Business 2020. Pour atteindre cet objectif, le Togo a déployé d'importants efforts de réforme dans les domaines de la création d'entreprise, de l'enregistrement de la propriété 
Version française étendue de l'auteur de : Kohnert, D. (2022): Togo. In : Albert K. Awedoba / Benedikt Kamski / Andreas Mehler / David Subudubudu (eds.), Africa Yearbook. Vol. 17 - Politics, economy and society South of the Sahara in 2020. Leiden \& Boston: Brill:, à paraitre

et de l'obtention de crédit. Cependant, l'Indice de liberté économique 2020 de la Heritage Foundation a classé le Togo toujours parmi les pays «majoritairement non libres » (rang 140 sur 100, score: 54,3, +3,8). 
Version française étendue de l'auteur de : Kohnert, D. (2022): Togo. In : Albert K. Awedoba / Benedikt Kamski /

Andreas Mehler / David Subudubudu (eds.), Africa Yearbook. Vol. 17 - Politics, economy and society South of the Sahara in 2020. Leiden \& Boston: Brill:, à paraitre

\section{Selected most current references and further reading (hyperlinked)}

AfDB (2020): Togo - African Economic Outlook. Abidjan: African Development Bank

Clarke, Richard (2020): Strengthening the climate resilience of cities through cross-border cooperation. OECD, 25 September 2020

Dogbe, Peter (2020): Togo: quel bilan de la première année de mandat pour les maires? rfi, $09 / 07 / 2020$

EIU (2020): Togo - Country Reports. London: Economist Intelligence Unit

EIU (2020): Democracy Index 2019. A year of democratic setbacks and popular protest. London

Freedomhouse (2020): Togo country report. Freedom in the World 2020. Washington D.C.

Kohnert, Dirk (2021): BTI -2022 Togo Country Report : political and socio-economic development, 2019-2020 [enhanced author's version]. Researchgate, 2021

Kuvo, Pierre-Claver (2020): Blanchiment d'argent et financement du terrorisme : Une société togolaise en affaire avec une entreprise Offshore à Singapour. CENOZO, Investigative Reporting in West Africa, September 20, 2020

Liberte Hebdo (2020): Togo - Dossier/Forces de défense et de sécurité du Togo : Inégalités dans les recrutements et nominations à des postes de responsabilités. Lomé: 27 Mai 2020

Mignanou Yawovi Amouzouvi, Koffi Sagna, Milohum Mikesokpo Dzagli, Sidéra Kodjovi Edjame, Agbéko Messanh Mohou (2019): Contribution of the Satellite Observation on the Analysis and the Forecast of the State of Air Pollution in TOGO. American Journal of Environmental Protection. Vol. 8, No. 1, 2019, pp. 31-38

Mo Ibrahim Foundation (2020) : Agendas 2063 \& 2030: Is Africa on track ? African Governance Report. London : Mo Ibrahim Foundation

OECD (2020) : Corona-policy country tracker. Togo. Paris : Organisation for Economic Cooperation and Development

Preuss, H.J. (2020) : Togo's dynasty lives on. ipg-journal, International Politics and Society, 28.02.2020

REDD+Togo (2018) : Etude sur les causes et consequences de la deforestation et la degradation des forets au Togo et identification des axes d'intervention appropies. Lomé : Republique Togolaise, 30.03.2018

Takouleu, Jean Marie (2020) : Togo: Fenix, Solergie and Moon join Cizo project to electrify villages. Afrik21, February 172020.

United Nations: World Happiness Report - 2020. Washington D.C.

US Dept. of State (2020): Togo - Country Reports on Human Rights Practices. Washington D.C.

World Bank (2020): Economic profile - Togo. Doing Business, 2020. Washington D.C. 
Version française étendue de l'auteur de : Kohnert, D. (2022): Togo. In : Albert K. Awedoba / Benedikt Kamski / Andreas Mehler / David Subudubudu (eds.), Africa Yearbook. Vol. 17 - Politics, economy and society South of the Sahara in 2020. Leiden \& Boston: Brill:, à paraitre

\begin{abstract}
Controversial constitutional and institutional reforms voted in May 2019 in parliament opened the way for President Gnassingbé to stand for a fourth and fifth term because the law does not apply retroactively. In February 2020, the President won again the disputed presidential elections and thus consolidated his power, assisted by the loyal army and security services. The outbreak of the Corona epidemic in Togo in March and the subsequent economic recession may have contributed to limit popular protest against the Gnassingbé regime. The human rights record of the government has improved, but remains poor. Yet, the international community followed a 'laissez faire' approach in the interests of regional stability. The economy dropped into recession due to the worldwide economic negative effects of the corona-crisis. The democracy index of the Economic Intelligence Unit, London, still rated Togo as an 'authoritarian regime'.
\end{abstract}

Résumé : Les réformes constitutionnelles et institutionnelles controversées votées en mai 2019 au Parlement ont ouvert la voie au président Gnassingbé pour se présenter pour un quatrième et un cinquième mandat parce que la loi ne s'applique pas rétroactivement. En février 2020, le président a remporté à nouveau les élections présidentielles disputées et a ainsi consolidé son pouvoir, assisté par l'armée fidèle et les services de sécurité. Le déclenchement de l'épidémie de Corona au Togo en mars et la récession économique qui a suivi a peut-être contribué à limiter les protestations populaires contre le régime de Gnassingbé. Le bilan du gouvernement en matière de droits de l'homme s'est amélioré, mais reste médiocre. Pourtant, la communauté internationale a suivi une approche de «laissez-faire» dans l'intérêt de la stabilité régionale. L'économie est entrée en récession en raison des effets négatifs économiques mondiaux de la crise corona. L'indice de démocratie de l'Economic Intelligence Unit, à Londres, classait toujours le Togo comme un «régime autoritaire».

Zusammenfassung : Umstrittene verfassungsrechtliche und institutionelle Reformen, die im Mai 2019 im Parlament verabschiedet wurden, eröffneten Präsident Gnassingbé den Weg für eine vierte und fünfte Amtszeit, da das Gesetz nicht rückwirkend gilt. Im Februar 2020 gewann der Präsident erneut die umstrittenen Präsidentschaftswahlen und festigte damit seine Macht, unterstützt von der loyalen Armee und den Sicherheitsdiensten. Der Ausbruch der Corona-Epidemie in Togo im März und die anschließende wirtschaftliche Rezession trugen dazu bei, den Protest der Bevölkerung gegen das Gnassingbé-Regime zu begrenzen. Die Menschenrechtsbilanz der Regierung hat sich verbessert, bleibt aber defizitär. Die internationale Gemeinschaft verfolgte trotzdem im Interesse der regionalen Stabilität einen Laissez-Faire-Ansatz. Die Wirtschaft geriet aufgrund der weltweiten wirtschaftlichen negativen Auswirkungen der Coronakrise in eine Rezession. Der Demokratieindex der Economic Intelligence Unit in London bewertete Togo weiterhin als "autoritäres Regime". 